\title{
GRAPE - GIS REPETITION USING AUDIO-VISUAL REPETITION UNITS AND ITS LEANRING EFFECTIVENESS
}

\author{
M. Niederhuber ${ }^{a, *}$, S. Brugger ${ }^{b}$ \\ ${ }^{a}$ ETH Zurich, Institut of Terrestrial Ecosystem, 8092 Zurich, Switzerland, monika.niederhuber@env.ethz.ch \\ ${ }^{\mathrm{b}}$ ETH Zurich, Institut of Terrestrial Ecosystem, 8092 Zurich, Switzerland, sarah.brugger@env.ethz.ch
}

\section{Commission VI, WG VI/4}

KEY WORDS: GIS, Teaching, Learning, Multimedia, Education, Training, Web based, Podcast

\begin{abstract}
:
A new audio-visual learning medium has been developed at the Department of Environmental Sciences at ETH Zurich (Switzerland), for use in geographical information sciences (GIS) courses. This new medium, presented in the form of Repetition Units, allows students to review and consolidate the most important learning concepts on an individual basis. The new material consists of: a) a short enhanced podcast (recorded and spoken slide show) with a maximum duration of 5 minutes, which focuses on only one important aspect of a lecture's theme; b) one or two relevant exercises, covering different cognitive levels of learning, with a maximum duration of 10 minutes; and c), solutions for the exercises. During a pilot phase in 2010, six Repetition Units were produced by the lecturers. Twenty more Repetition Units will be produced by our students during the fall semester of 2011 and 2012.

The project is accompanied by a 5-year study $(2009-2013)$ that investigates learning success using the new material, focussing on the question, whether or not the new material help to consolidate and refresh basic GIS knowledge. It will be analysed based on longitudinal studies. Initial results indicate that the new medium helps to refresh knowledge as the test groups scored higher than the control group. These results are encouraging and suggest that the new material with its combination of short audio-visual podcasts and relevant exercises help to consolidate students' knowledge.
\end{abstract}

\section{KURZFASSUNG:}

Am Departement Umweltnaturwissenschaften der ETH Zürich (Schweiz) wird ein neues, audio-visuelles Lernmedium für die Kurse im Bereich Geographische Informationswissenschaften (GIS) entwickelt. Das neue Medium besteht aus einzelnen Repräsentationseinheiten, welche den Studierenden erlauben, die wichtigsten GIS-Grundlagen eigenständig zu wiederholen und zu festigen. Das neue Material besteht aus: a) einem kurzen Podcast (aufgezeichnete und besprochene PowerPoint Präsentation) mit einer maximalen Länge von 5 Minuten, welcher nur einen wichtigen Aspekt eines Vorlesungsthemas beinhaltet; b) darauf aufbauend, ein oder zwei Übungsaufgaben, die verschiedene, auch höhere, Lernzielstufen abdecken und maximal 10 Minuten Bearbeitungszeit benötigen; und c), Lösungen für die Übungsaufgaben. Während einer Pilotphase in 2010 wurden sechs Repetitionseinheiten seitens der Dozierenden entwickelt. Weitere 20 Repetitionseinheiten werden im Herbstsemester 2011 und 2012 von unseren Studierenden produziert.

Das Projekt wird von einer 5-jährigen Wirksamkeitsstudie (2009 - 2013) begleitet, welche den Lernerfolg des neuen Mediums untersuchen soll. Im Zentrum steht dabei die Frage, ob die audio-visuellen Repetitionseinheiten hilfreich sind, die GIS-Grundlagen zu festigen bzw. aufzufrischen. Die Analysen basieren auf Längsschnittstudien. Erste Ergebnisse zeigen, dass die Testgruppen besser abgeschnitten haben als die Kontrollgruppen. Das Ergebnis ist ermutigend und weist darauf hin, dass das neue Medium hilfreich ist bei der Festigung des Wissens.

\section{INTRODUCTION}

We offer four GIS courses at the Department of Environmental Science at ETH Zurich, two at the bachelor level and two at the master level. Between the main GIS bachelor course "Introduction into geo-information science and technology" and the main GIS master course "Spatial analysis and modeling", there is a time gap of at least 14 month, and in some cases, up to three years. Due to this time gap, we observed a very heterogeneous level of knowledge in GIS at the beginning of our master course. Up to now, we have tried to solve this problem by doing a fast group repetition of the most important learning objectives of the bachelor course within the first two hours of the master course. Unfortunately, this type of repetition neither considers the students' individual state of knowledge, learning behaviour and speed, nor does it establish a higher and more homogeneous entrance level for the master course. Within the project "GRAPE", financially supported by the Innovedum fund (www.innovedum.ethz.ch) of the ETH Zurich, we have been developing a new method and accompanying materials for the students to independently refresh their knowledge before the start of the master's course.

\footnotetext{
* Corresponding author.
} 
Additionally, this new approach should also be applicable as an extra study aide for the bachelor course. The project is accompanied by a 5-year study that investigates learning effectiveness using the new material.

This paper is composed of two main sections: the conceptual design for creating the audio-visual Repetition Units will be described first, followed by an overview and initial results of the learning effectiveness study. The paper ends with a conclusion and outlook.

\section{GIS REPETITION USING AUDIO-VISUAL REPETITION UNITS}

\subsection{Conceptual design}

Within our main GIS bachelor course, the learning objectives of a lesson are described using the Anderson et al. (2001) taxonomy. This taxonomy defines the following six cognitive levels: (1) remembering, (2) understanding, (3) applying, (4) analyzing, (5) evaluating and (6) creating. It is important for us that even our bachelor students can reach higher cognitive levels such as analyzing, evaluating, or creating. A fundamental requirement of the new material was to ensure that also these higher cognitive levels may be reached and/or re-established. This suggests that not only should read-only material be provided, but also more ambitious interactive exercises. Furthermore, we think that a mixture of learning materials is more attractive and helps to maintain attention.

After various discussions about "what might be attractive material", we decided to produce Repetition Units consisting of a mix of materials, including (a) enhanced podcasts (audiovisual presentation), (b) one or two relevant exercises, and (c) solutions. The basis of this material is the learning content of the bachelor course, which we will split into sub sections (see Figure 1).

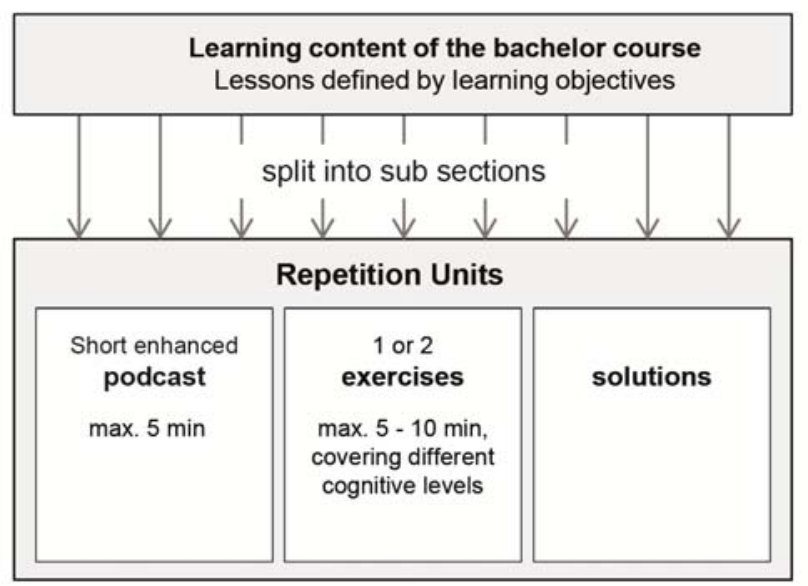

Figure 1. Conceptual design of the Repetition Units

(a) Enhanced podcasts (audio-visual presentation): Important aspects of a lecture's topic will each be audiovisually explained with the help of an enhanced podcast (recorded and spoken slide show) with a maximum duration of 5 minutes. The content and the layout of the podcasts correspond directly to the lectures, in order to facilitate learning and knowledge acquisition. With the podcasts, the students can refresh and/or consolidate their theoretical understanding of a topic.
Each podcast consists of three parts: a title slide, the main body and the final slide. The title slide of each podcast starts with a jingle to create attention to the podcast and audio-visualizes the title, the name of the author(s) and the copyrights. The main part contains all the slides and explains a specific, well defined topic, ending with the references. Each podcasts ends with a final slide, briefly outlining the details of the project. In order to achieve homogeneity within our Repetition Pool, the title and final slide will always be produced by the same person (i.e. lecturer) for all podcasts. The students will be responsible for the production of the main body and the references.

For the distribution of our podcasts in different audio and video formats, we decided to use the established technical infrastructure of the Multimedia Portal of ETH Zurich (http://www.multimedia. ethz.ch).

(b) Exercises: One or two relevant exercises will be developed, covering different levels of learning objectives, with a maximum duration of 10 minutes. By completing the exercises, students can reinforce theoretical knowledge using realistic examples and hopefully by doing so achieve a higher cognitive level.

As a medium, we use Word templates, converted afterwards into pdf. This is an efficient way to produce exercises which may also contain drawing tasks.

(c) Solutions: With the solutions, students can individually check the results of the exercises, allowing them to work independently from a class or teacher.

During a pilot phase in 2010, six Repetition Units were produced by the lecturers and a technical workflow was developed. These units were used and evaluated by bachelor students during the 2010 fall semester and by master students during the 2011 spring semester. In summary, the students accepted and welcomed this new learning medium to supplement their regular course work. Based on this positive feedback, we decided to produce twenty more Repetition Units (see section 2.2).

\subsection{Two Approaches: "Students as Producers" and "Students as Users"}

We have planned to produce twenty more Repetition Units, created by the bachelor students within the GIS basic course during the fall semesters of 2011 and 2012. Therewith, the bachelor students have two roles within this project: a) they are the producers of the Repetition Units, and b) they will be the users as well (see figure 2).

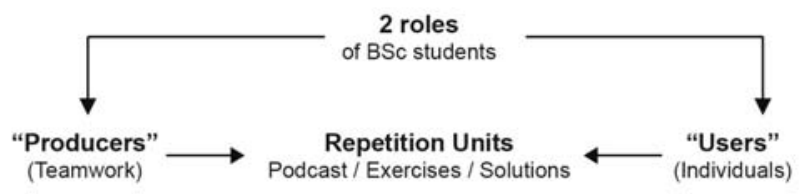

Figure 2. Roles of BSc.-Students within GRAPE 
(a) "Students as Producers": Due to the active participation of our students within the project, it has been necessary to work out a simple approach for the development of the material. We assume that all students have their own computer, but we need an approach, working under the both operating systems "Windows" and "Mac". Furthermore, the software used by the students has to be free of charge. After testing different software products, we decided to record the enhanced podcasts using "ProfCast", which can be used without a license key for 15 days (ProfCast, 2010). For the final cut, we will use "Final Cut Studio" (Apple, 2009).

In order to implement this approach, a technical and didactical guideline, as well as a workflow for the production of the podcasts and exercises has been written. Templates have been prepared to maintain homogeneity within our Repetition Pool. The titles of the relevant Repetition Units will be provided by the lecturers, including a short summary and a list with the learning objectives. The students will produce the Repetition Units in small groups of 4 or 5 members to minimize the students' work load.

At the beginning of the semester, the lecturer will introduce the project and the steps of the workflow, and assign the relevant topics to the students. Thereafter, the students familiarize themselves with the topic through the use of literature and the web and assign the different tasks within their group. The students can now start to prepare drafts of the PowerPoint slides, slide manuscripts, and exercises. The drafts will be reviewed and discussed with the lecturers. Afterwards, it is the students' task to embed the lecturers' comments and corrections into their material and to produce a second version of the slides, manuscript, and exercises and to prepare the solutions of the exercises. This version will be reviewed and discussed again with the lecturers. In the next step, the students will revise their materials and record their podcast. The final products will be delivered to the lecturers and reviewed. If the products have reached the required standard, the lecturers will integrate them into the Repetition Pool; otherwise, the students have to improve their material. During the whole process, the lecturers supervise the student and consult them on technical and didactical questions.

With the "Students as Producer" approach, the students' can gain core skills in addition to GIS-specific ones (in German: "Überfachliche Kompetenz", see Maag Merki and Schuler, 2003), such as:

- $\quad$ project management and planning abilities;

- teamwork, whereby the students can obtain communicative and social experiences;

- $\quad$ production in addition to usage of multi-media;
- didactical-psychological competences by thinking about questions such as "how can I explain something?", "what is the best way to present something using multi-media"?, etc.;

As the organizers of the project, we hope that the students' will be motivated to create the Repetition Units since their work will be included in the Repetition Pool and can benefit other students. (b) Students as Users: Students' access to the Repetition Units is provided through the learning platform "Moodle" (http://moodle.org), currently password protected. On Moodle, every unit is listed along with a short summary as well as a list of learning objectives. Access to the podcast, exercises and solutions is given via a link within Moodle.

The students can independently check their current level of GIS knowledge using the short summary or the list with learning objectives. With it, they can identify gaps. Since the Repetition Units are modularly designed and therefore independent from each other, the students can choose the relevant units to study, in order to re-activate or consolidate their knowledge. After the first study cycle, it is the students' responsibility to check if they have mastered the expected learning outcomes (see figure $3)$.

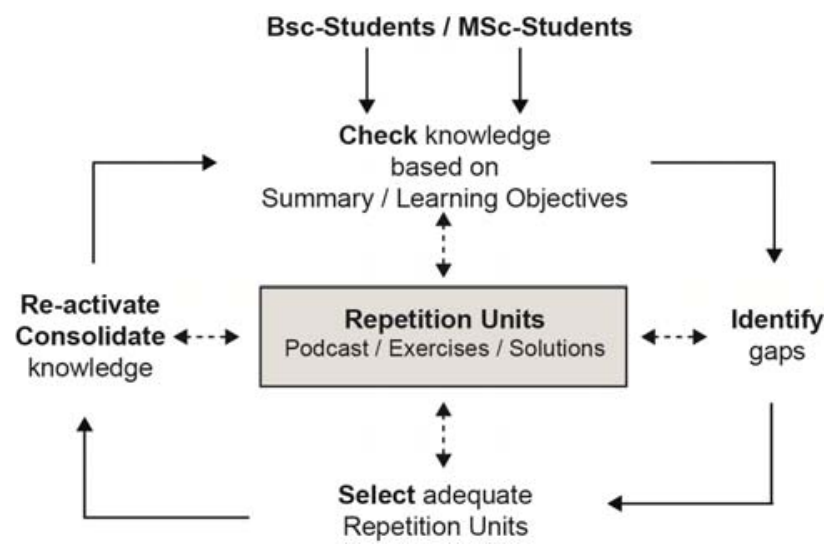

Figure 3. "Study cycle" of the Repetition Units

The advantages of the Repetition Units are that the students are encouraged to study independently and in a self-organized manner. The students are free to decide whether they will watch the podcast first and do the exercises afterwards or vice versa, or only use one of the offered media. Furthermore, studying can take place at the students own pace and is time and location independent.

\subsection{Project evaluation - first results}

An initial didactical and technical evaluation of our project was carried out in March / April 2011 by two members of the ETH Educational Development and Technology (LET) unit and by a senior researcher from the Chair of Educational Media and Knowledge Management from the University of DuisburgEssen (Germany). From their evaluation, they believe that the Repetition Units are a simple and fast medium for the students to get a good overview of a clearly defined topic. The podcasts' length of 3 to 5 minutes seems to be ideal and the exercises are clearly structured. Furthermore, the evaluators gave us additional suggestions, such as: (1) to use a longer jingle at the beginning to better direct attention to the podcast; (2) to improve the podcasts by including animations; (3) to speak more freely and to include verbal emotions; (4) to sum up each podcast with the most important take-home messages. We will consider these remarks as far as possible on the revised versions of the podcast and pass these remarks on to the students.

A students' evaluation was carried out at the end of the fall semester 2010 with 21 bachelor students and at the spring 
semester 2011 with 10 master students using a questionnaire. Additionally, we had individual - not planned - discussions. The evaluation shows that most of the students used this new medium on personal computers at home or at ETH. Up to now, only one student was watching the podcasts on the way with a hand-held medium. The students welcomed this new medium as an additional learning source and found the material, both podcasts and exercises, helpful for the preparation of the exam. The students appreciated the duration and right amount of information within a podcast.

These first results are very encouraging to continue and to improve our project.

\section{LEARNING EFFECTIVENESS STUDY}

In our learning effectiveness study, we investigate the ability of students to give concrete statements about the impact of Repetition Units.

There are a number of different studies that investigate the use of podcasts for students in an academic environment. Chan and Lee (2005) describe the application of podcasting in helping to reduce student anxieties regarding information technology. Edirisingha and Salmon (2007) investigated the impact of podcasting on student learning in higher education and found that podcasts can improve their engagement. Evans (2007) found that students believe that podcasts are more effective and efficient for delivering content than textbooks. Khechine et al. (2009) describe the effectiveness of podcasting technology for teaching and learning. They investigated an online course with a questionnaire and found out that the students who used podcasts demonstrated a deeper learning and a greater satisfaction. Badowski and Collage (2009) found that podcasts help students improve studying for examinations.

Most of these studies are based on questionnaires and selfevaluations, or on a single examination or test. Our project involves a longitudinal study that analyzes learning success over five years, (2009 - 2013), focusing on the following questions:

Q1: Can the Repetition Units help to consolidate GIS knowledge within the bachelor Course?

This first question compares the benefit of the Repetition Units with the conventional tools like PowerPoint slides, notes and textbooks. Both groups (test and control group) received the same information; in addition to the conventional tools, the test group had access to the Repetition Units.

Q2: How much previous knowledge do the students have when starting the master course in comparison to the end of the bachelor course?

The second question compares the knowledge at the end of the bachelor's course with the knowledge at the beginning of the master's course. The time gap between these two courses can be at least 14 months and therefore the level of knowledge is normally very heterogeneous.

Q3: Is the new material better at refreshing basic GIS knowledge at the beginning of the master's course, compared to our conventional method?
The third question investigates whether the Repetition Units help to better refresh the basic knowledge or whether a common repetition in the class is more effective. The aim would be a more homogeneous and higher entry level for the master's course without the need to spend time repeating subjects during the class.

\subsection{Study design}

This section describes the methodological structure of the study. To be able to make statements about the effectiveness of the Repetition Units, an observational study was chosen. The observations are repeated events of the same variable over a longer period. Therefore, the learning success will be analyzed based on these longitudinal studies. Test series have been developed, and every semester, the students will be randomly allocated to either a test or control group. The test group has access to the Repetition Units and uses this new material, while the control group uses only the conventional material such us textbooks and lecture notes. The division into test and control group is statistically independent and unbiased.

3.1.1 Test environment: Tests are arranged during the semester in the classrooms. It is a written test with different types of questions including short answer and longer open questions as well as simple calculations. The students have a time limit of 30 minutes and no tools are available during the test.

3.1.2 Samples and procedure: Most of the participants were students from the Department of Environmental Science from the ETH and were between 21 and 25 years old. The number of females and males was roughly the same in each group.

Q1: The study sample for the first question was made up of bachelor students enrolled in the GIST (Introduction into geoinformation science and technology) course. This course is offered in the 5th semester as an optional course. The following analyses are based on the fall semester 2010 with 41 students 22 female and 19 male. All the participants had the same level of understanding of GIS.

We were not allowed to influence the official examination at the end of the semester with our study. Therefore, we carried out our test one week before the final examination. The test group (21 students) was invited to use the Repetition Units' through Moodle. The control group (20 students) studied the topics with conventional materials.

Q2: To investigate the GIS knowledge of the students at the beginning of the master semester this study compares the final bachelor examination with the test at the beginning of the master's course. This investigation was conducted in 2009 and 2010. For the following analysis we used only the questions which were the same in all the tests.

Six students who took part in the 2007 exam also took part in the 2009 test. Ten students from the 2007 exam also took part in the 2010 test and finally a further four students who took part in the 2008 exam also completed the test in 2010 (see table 2).

Q3: The sample for the third question was comprised of master students from the SAM (Spatial analysis and modeling) course. This is an optional course for the master degree in forest and 
landscape management. The following analyses are based on the spring semester 2011 comprised of 17 students -6 female and 11 male.

At the beginning of the master's course all the students had to complete our test in order to produce a baseline. Afterward we randomly divided the students into test and control groups. The test group (8 students) was invited to use the Repetition Units through Moodle to repeat the GIS topics. The control group repeated the same subjects with the teacher in the classroom. After one week, all students had to repeat the test again.

3.1.3 Sample Size: For the second and the third questions the sample size (n) was relatively small. Due to the small sample sizes in certain years, the strength of the tests used was not very high. This may explain why the individual tests did not reveal significant differences between the groups. We will therefore repeat our test over several years to combine the evidence of several tests.

3.1.4 Analysis Tools: In the current study the collected data are available as paired sample data. This is because students were tested twice. A two-tailed test was chosen to test the null hypothesis. The paired t-test helps to examine if there is a statistical difference between two groups. As an alternative, we used a Wilcoxon two-sample test, which does not assume normal distribution of data. All statistical tests were performed with a p-value of 0.05 . The statistical analyses were conducted using the software packages S-Plus (TIBCO Software, 2010) and SAS (SAS, 2008).

\subsection{Preliminary results}

Preliminary results indicate that the new medium helps to refresh knowledge for both the bachelor's course and the master's course. In both cases the test group scored higher than the control group. These results are encouraging and suggest that the new material with its combination of short audio-visual podcasts and relevant exercises is attractive and helpful to consolidate knowledge.

Q1: The first research hypothesis was tested using a Wilcoxon two-sample test.

Table 1 shows the average scores across students of the test group who studied with the Repetition Units and the control group with the conventional material.

\begin{tabular}{|l|l|l|}
\hline $\begin{array}{l}\text { average scores across } \\
\text { students in the test } \\
\text { group }(\mathrm{n}=21)\end{array}$ & $\begin{array}{l}\text { average scores across } \\
\text { students in control } \\
\text { group }(\mathrm{n}=20)\end{array}$ & p-value \\
\hline 11.4 & 8.2 & 0.0013 \\
\hline
\end{tabular}

Table 1. Average scores across students in test and control group at the end of the bachelor course (fall semester 2010)

The maximum score of this test was 16 points. The test group scored on average 11.4 points, compared to 8.18 for the control group. This means that the test group scored around $20 \%$ higher than the control group.

Based on the result of the Wilcoxon test with a p-value of 0.0013 , we can state that there is a significant difference between the students who used the Repetition Units and those who did not.

This first result indicates that the Repetition Units help to consolidate GIS knowledge. This test will be repeated in fall 2011 and 2012.

Q2: The second question was tested using a paired T-Test. The results of the comparison of the same student between the final exam in the bachelor's course (2007 and 2008) with the test at least 14 months later in the master's course $(2009,2010)$ are presented in the table below. The maximum possible score within this test was 33 .

\begin{tabular}{|c|c|c|l|l|l|r|c|}
\hline $\mathrm{G}$ & $\mathrm{n}$ & \multicolumn{2}{|c|}{ Bachelor Exam } & \multicolumn{2}{c|}{ Master Test } & $\Delta$ & p-value \\
\hline & & Year & Score & Year & Score & & \\
\hline 1 & 6 & 2007 & 20.8 & 2009 & 10.8 & -10.0 & 0.0027 \\
\hline 2 & 10 & 2007 & 21.0 & 2010 & 13.5 & -7.5 & 0.0005 \\
\hline 3 & 4 & 2008 & 23.4 & 2010 & 12.3 & -11.1 & 0.0254 \\
\hline
\end{tabular}

$\mathrm{G}=$ Group; $\mathrm{n}=$ Sample size; Score: average score across students $\Delta=$ Score differences: master test minus bachelor exam

Table 2. Average scores across students at the end of the bachelor and start of the master course, and its differences

The evaluation shows that the students performed significantly better in the exam administered directly after completion of the bachelor course (2007 and 2008). The students achieved substantially less points on the test administered at the beginning of the master semester (2009 and 2010). The results show a relatively large drop in retained knowledge, which confirms our assumptions.

Q3: To answer the third question, two tests were given at the first two weeks of the spring semester 2011. The first test was needed to show if there is a significant difference between the test and the control group before the repetition. As we can see in table 3, both groups reached around the same number of scores (test group: 5.53; control group: 5.81; maximum possible score: 16) and, there is no significant difference between these two groups ( $\mathrm{p}=0.5566$, Wilcoxon Two-Sample Test, two-sided).

\begin{tabular}{|l|c|c|}
\hline & $\begin{array}{l}\text { Average scores } \\
\text { across students in } \\
\text { test group (n=8) }\end{array}$ & $\begin{array}{l}\text { Average scores } \\
\text { across students in } \\
\text { control group } \\
(\mathrm{n}=9)\end{array}$ \\
\hline $\begin{array}{l}\text { First test: } \\
\text { before Repetition }\end{array}$ & 5.5 & 5.8 \\
\hline $\begin{array}{l}\text { Second test: } \\
\text { after Repetition }\end{array}$ & 8.6 & 7.4 \\
\hline Difference & +3.1 & +1.6 \\
\hline
\end{tabular}

Table 3. Average scores across students in test and control group, before and after the repetition

In the second test, the students who used the Repetition Units obtained in average 8.59 scores while the students in the control group archived in average 7.42 scores. Strictly speaking there were no significant differences $(\mathrm{p}=0.1169)$ between groups with respect to the scores obtained in both tests. The scores for the second test were better for all students. However, the one-sided Wilcoxon Two-Sample test may suggest that the score improvements were slightly better in the test group $(\mathrm{p}=0.0586)$. 
One should take into account that the strength of the test is rather small because of the small sample sizes. This test will be repeated in fall 2012 and 2013, so that a comparison of three years will be possible.

\section{CONCLUSIONS AND OUTLOOK}

The feedback from our students is very encouraging and suggests that this new learning material with its combination of short enhanced podcasts and relevant exercises are attractive and helpful to consolidate and to refresh knowledge. The bachelor students can use the material, both in parallel with the lessons and in preparation of the final exam. With it, we expect an improvement of the students' scores of the final exam. However, this assumption can only be tested towards the end of our project.

Based on our test results, there is a relatively large drop of knowledge between the end of the bachelor course and the beginning of the master course. The previous knowledge of the master students can be improved using this new material, but in comparison to the bachelor course, we could not yet measure a significant difference between the test and the control group. However, with the new medium the students can independently refresh their knowledge and, after having finished the project, it is no longer necessary for us to sum up basic knowledge in class. The free time can be used for advanced and more exciting topics within spatial analysis and modeling.

Our students can also use the Repetition Units to brush up GIS knowledge within our GIS case study course. Furthermore, we plan to produce the material in such a way that it might be used as open educational resources in the future.

During August / September 2011, we have a test phase with two students, producing one Repetition Unit. Besides the production of the Repetition Unit, they have to evaluate the technical and didactical guidelines as well as the detailed workflow. Their feedback will be integrated into the project. This test phase is very important for us to collect first hand experiences with the students' handling of our information, guidelines, etc. It will reveal difficulties and give us an indication of the time and workload. Within the fall semester of 2011 and 2012, the students will be given the assignment to develop the Repetition Units and we will continue our learning effectiveness study.

\section{REFERENCES}

Anderson, L.W., Krathwohl, D.R., Airasian, P.W., Cruikshank, K.A., Mayer, R.E., Raths, J. et al., 2001. A taxonomy for learning, teaching and assessing. A revision of Bloom's taxonomy of educational objectives. New York.

Apple, 2009. Final Cut Studio. Version 3. Appel Inc.

Badowski, R., College, T., 2009: The Effectiveness of Podcasting on Achievement in Principles of Accounting. 2009 In: Proceedings of the 2009 ASCUE Summer Conference. June 14-18, 2009, North Myrtle Beach, South Carolina. http://www.ascue.org/files/proceedings/2009/p32.pdf (accessed 23Aug. 2011).

Chan, A., Lee, M., 2005. An MP3 A Day Keeps The Worries Away: Exploring the use of podcasting to address preconceptions and alleviate pre-class anxiety amongst undergraduate information technology students. Paper presented at the Student Experience Conference, 5-7 September 2005, Wagga Wagga, New South Wales. http://citeseerx. ist.psu.edu/viewdoc/summary?doi=10.1.1.115.1023 (accessed 23 Aug. 2011).

Edirisingha, P., Salmon, G., 2007. Pedagogical models for podcasts in higher education. In: EDEN conference, 13-16 June 2007, Naples. http://podcastingforpp.pbworks.com/f/palithra. pdf (accessed 23 Aug. 2011).

Evans, C., 2008. The effectiveness of m-learning in the form of podcast revision lectures in higher education. In: Computers \& Education. Vol. 50, Issue: 2, Elsevier, Pages: 491-498.

http://www.purdue.edu/discoverypark/PLM/MCCDE/curriculu $\mathrm{m} /$ modules/podcast/m-learning_podcasts.pdf (accessed 23 Aug. 2011).

Khechine H., Lakhal, S., Pascot, D., 2009. A Comparative Study on the Effectiveness of Podcasting in Distance Learning. In: ICIW '09 Proceedings of the 2009 Fourth International conference on Internet and Web Applications and Services. IEEE Computer Society Washington, DC, USA. $\mathrm{http} / /$ ieeexplore.ieee.org/stamp/stamp.jsp?tp=\&arnumber $=5072$ 583 (accessd 23 Aug. 2011).

Maag Merki, K., Schuler, P. (Hrsg.), 2003. Überfachliche Kompetenzen. Schriftenreihe zu „Bildungssystem und Humanentwicklung“. Berichte aus dem Forschungsbereich Schulqualität \& Schulentwicklung.

ProfCast, 2009: ProfCast for Windows. Version 1.0.59.545 Humble Daisy, Inc.

SAS, 2008. SAS 9.2 for Windows. SAS Institute Inc. Cary, NC, USA.

TIPCO Software, 2010. Spotfire $S+\circledR 8.2$ for Windows, TIBCO Software Inc.

\section{ACKNOWLEDGMENT}

The project „GRAPE - GIS repetition using audio-visual podcasts elements and its learning effectiveness study" is financially supported by the Innovedum fund of the ETH Zurich (www.innovedum.ethz.ch).

Many thanks to Thomas Korner and Andreas Reinhardt from the Educational Development and Technology (LET) unit of the ETH Zurich for their technical support and valuable feedback.

Many thanks to Mandy Rohs from the Chair of Educational Media and Knowledge Management from the University of Duisburg-Essen (Germany) for her didactical support and invaluable expertise.

Furthermore, we would like to thank our colleague Daniel Mandallaz for his statistical support. 